\title{
Utilización analítica del Contorno de bloqueo restringido por las tolerancias en engranajes cilíndricos exteriores
}

\author{
Analytical use of blocking contour restricted by tolerances in external \\ cylindrical gears \\ Robert Hernández Ortega, Feliberto Fernández Castañeda \\ Departamento Ingeniería Mecánica, Universidad Central Marta Abreu de Las Villas, Santa Clara, Cuba \\ robertho@uclv.edu.cu
}

\begin{abstract}
Resumen- Los Contornos de Bloqueo muestran al diseñador de engranajes el conjunto de valores de los coeficientes de corrección, que cumple con las limitaciones geométricas de socavado, factor de recubrimiento, interferencia y espesor del diente en el diámetro exterior. En este trabajo se perfecciona este método, considerando para su construcción las tolerancias de la distancia entre centros, el diámetro exterior y la holgura lateral entre los perfiles de los dientes. De esta manera se garantiza el cumplimiento de las restricciones mencionadas, aun con las combinaciones más desfavorables de las tolerancias, lo que mejora la calidad del diseño de estas transmisiones.
\end{abstract}

Palabras clave - Contorno de Bloqueo, coeficientes de corrección, tolerancias, engranajes

Abstract- Blocking Contours offers the gear designer the addendum modification coefficients values that fulfills the geometric limitations of undercut, transverse contact ratio, interference and tooth pointed. In this work this method is improved, considering in the contour's construction the tolerances of the center distance, the tip diameter and the backlash between teeth profiles. Thus, the achievement of the mentioned geometric limitations is guaranteed, even with the most unfavorable combinations in the tolerances, what improves the design quality of these transmissions.

Key Word - Blocking Contour, addendum modification coefficients, tolerances, gear

\section{INTRODUCCIÓN}

Los Contornos de Bloqueo son un método gráfico, cuyos principios fundamentales para su construcción fueron desarrollados por el ingeniero ruso Israel Bolotovski [1] hace más de 60 años. Esta técnica consiste en la introducción en un sistema de coordenadas (Fig. 1): coeficiente de corrección de la rueda $\left(\mathrm{x}_{2}\right)$ versus coeficiente de corrección del piñón $\left(\mathrm{x}_{1}\right)$, las curvas que definen las restricciones geométricas siguientes:
1. Factor de recubrimiento.

2. Espesor del diente en el diámetro exterior del piñón.

3. Interferencia del diámetro exterior de la rueda con el pie del diente del piñón.

4. Interferencia del diámetro exterior del piñón con el pie del diente de la rueda.

5. Socavado en el piñón.

6. Socavado en la rueda.

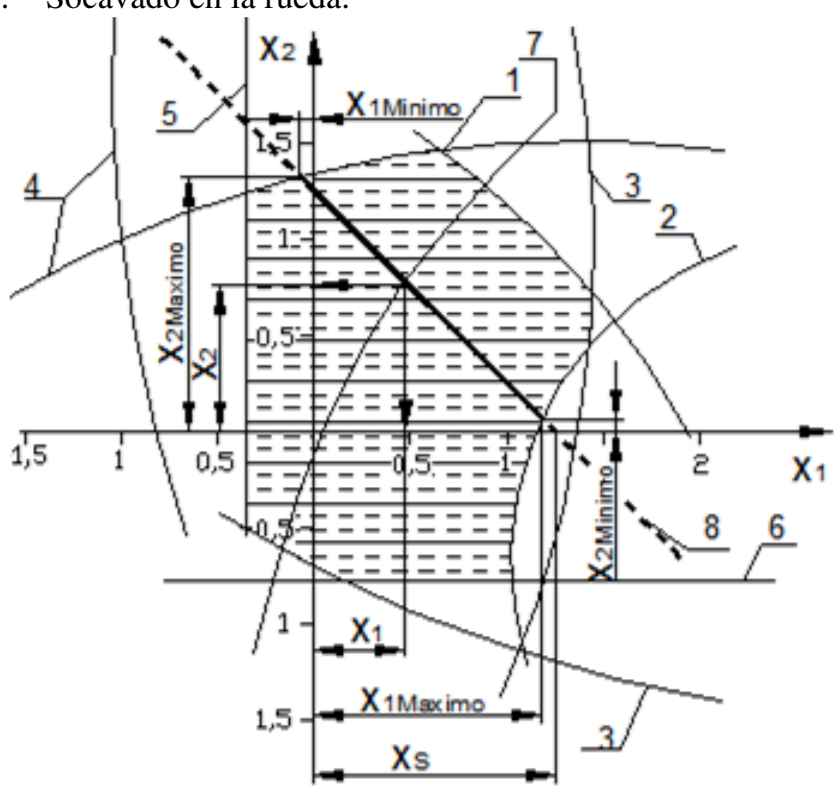

Figura 1. Contorno de Bloqueo

El conjunto de valores de $\mathrm{x}_{1} \mathrm{y}_{2}$ encerrados por estas curvas delimita la zona de existencia o dominio, de la transmisión definida por los números de dientes del piñón $\left(\mathrm{z}_{1}\right)$ y la rueda $\left(\mathrm{z}_{2}\right)$, el ángulo de inclinación del diente $(\beta)$ y los parámetros de la herramienta.

La introducción de las curvas en el área gráfica se realiza a partir de las ecuaciones de cada restricción geométrica 
expresadas para módulo unitario. La condición de socavado en su forma más general es la siguiente [1]:

$$
\mathrm{x}_{1,2} \geq \mathrm{h}-\frac{\mathrm{z}_{1,2} \cdot \operatorname{sen}^{2}\left(\alpha_{\mathrm{t}}\right)}{2 \cos (\beta)}
$$

Dónde:

$\propto_{\mathrm{t}} \rightarrow$ Ángulo de presión de la herramienta en el plano frontal:

$$
\begin{aligned}
& \propto_{\mathrm{t}}=\tan ^{-1}\left(\frac{\tan (\alpha)}{\cos (\beta)}\right) \\
& \mathrm{h}=\mathrm{ha}^{*}+\mathrm{c}^{*}-\rho^{*}+\rho^{*} \operatorname{sen}(\alpha)
\end{aligned}
$$

Parámetros de la herramienta:

ha $^{*} \rightarrow$ Coeficiente de altura de cabeza.

$\mathrm{c}^{*} \rightarrow$ Coeficiente de holgura radial.

$\rho^{*} \rightarrow$ Radio de redondeo.

$\alpha \rightarrow$ Angulo de presión.

El factor de recubrimiento se obtiene por la ecuación [1]:

$$
\varepsilon_{\propto}=\frac{1}{2 \cdot \pi}\left[\begin{array}{c}
\mathrm{z}_{1}\left(\tan \left(\alpha_{\mathrm{a} 1 \mathrm{t}}\right)-\tan \left(\alpha_{\mathrm{wt}}\right)\right)+ \\
\mathrm{z}_{2}\left(\tan \left(\alpha_{\mathrm{a} 2 \mathrm{t}}\right)-\tan \left(\alpha_{\mathrm{wt}}\right)\right)
\end{array}\right] \geq \varepsilon_{\alpha \text { Mínimo }}
$$

Donde:

$\alpha_{w t} \rightarrow$ Ángulo de presión en el diámetro primitivo medido en el plano frontal.

$\varepsilon_{\propto \text { Mínimo }} \rightarrow$ Factor de recubrimiento mínimo

$\alpha_{\mathrm{at}} \rightarrow$ Ángulo de presión en el diámetro exterior medido en el plano frontal, que se obtiene de la ecuación:

$$
\tan \left(\alpha_{\mathrm{at} 1,2}\right)=\frac{\sqrt{\mathrm{da}_{1,2}^{2}-\mathrm{do}_{1,2}^{2}}}{\mathrm{do}_{1,2}}
$$

do $1,2 \rightarrow$ Diámetros de las circunferencias base.

$\mathrm{da}_{1,2} \rightarrow$ Diámetros exteriores de las ruedas.

El espesor mínimo del diente en el diámetro exterior de las ruedas, medido en el plano normal [1]:

$$
\begin{aligned}
\operatorname{Sa}_{1,2}=\mathrm{da}_{1,2} \operatorname{Cos}(\beta)\left(\frac{\frac{\pi}{2}+2 \mathrm{x}_{1,2} \operatorname{Tan}\left(\propto_{\mathrm{t}}\right)}{\mathrm{z}_{1,2}}+\operatorname{Inv}\left(\propto_{\mathrm{t}}\right)\right. \\
\left.-\operatorname{Inv}\left(\propto_{\mathrm{a} 1,2 \mathrm{t}}\right)\right) \geq \operatorname{Sa}_{1,2 \text { Mínimo }}
\end{aligned}
$$

La condición de no interferencia se expresa por la siguiente inecuación [1]:

$$
\begin{aligned}
& \mathrm{do}_{1,2} \tan \left(\alpha_{\mathrm{wt}}\right)-\mathrm{do}_{2,1}\left(\tan \left(\alpha_{\mathrm{at} 2,1}\right)-\tan \left(\alpha_{\mathrm{wt}}\right)\right) \\
& \geq \mathrm{do}_{1,2} \tan \left(\alpha_{\mathrm{t}}\right)-2 \frac{\left(\mathrm{ha}^{*}+\mathrm{c}^{*}-\rho^{*}+\rho^{*} \operatorname{sen}(\alpha)-\mathrm{x}_{1,2}\right)}{\operatorname{sen}\left(\alpha_{\mathrm{t}}\right)}
\end{aligned}
$$

La utilización práctica del Contorno de Bloqueo se sustenta en el hecho de que una transmisión con un valor de corrección sumaria $\left(\mathrm{x}_{\mathrm{s}}\right)$ dado, se representa en el sistema de coordenadas $\mathrm{x}_{2}=\mathrm{f}\left(\mathrm{x}_{1}\right)$ como una recta inclinada a 45 grados (ver recta 8 en la Fig. 1), definida por la ecuación [1]:

$\mathrm{x}_{2}=\mathrm{x}_{\mathrm{s}}-\mathrm{x}_{1}$

Los puntos limitados por la intersección con los bordes del contorno ( $\left.\mathrm{x}_{1 \text { Mínimo, }}, \mathrm{x}_{2 \text { Máximo }}\right)$ y $\left(\mathrm{x}_{1 \text { Máximo, }}, \mathrm{x}_{2 \text { Mínimo }}\right)$ definen el intervalo de valores de los coeficientes de corrección que cumplen con todas las limitaciones geométricas. Debido a que el conjunto de combinaciones posibles entre los puntos señalados resulta demasiado amplio, se introducen en el área gráfica curvas que constituyen indicadores de calidad en el trabajo de las ruedas. Así por ejemplo, la curva 7 en la Fig. 1 representa la igualdad del deslizamiento específico entre los dientes.
De esta forma, mediante el método de los Contornos de Bloqueo, resulta fácil comprobar o asignar los coeficientes de corrección de las ruedas. A pesar de ello, en la práctica, a criterio de este autor, esta técnica no alcanzó una amplia utilización por las razones siguientes:

1. Se necesita disponer del Contorno de Bloqueo, con la combinación exacta de números de dientes y ángulo de inclinación del diente de la transmisión que se diseña. Según Bolotovski una alternativa de solución a este problema, es utilizar la combinación de dientes inmediata inferior, sin embargo, esto evidentemente aumenta el error de la síntesis. Por otro lado, resultaba prácticamente imposible disponer de Contornos de Bloqueo para todas las combinaciones posibles de números de dientes y ángulo de inclinación del diente.

2. La construcción del contorno para cada combinación de números de dientes y ángulo de inclinación del diente de la transmisión que se diseña, es una tarea compleja, que exige del diseñador un tiempo y esfuerzo importantes.

El estado actual de desarrollo de los medios informáticos permite eliminar las limitaciones mencionadas del método. Así, la construcción automatizada de los Contornos de Bloqueo posibilita la obtención de la zona válida de los coeficientes de corrección, para cualquier combinación de números de dientes y parámetros de la herramienta, de una manera rápida y sencilla. En este sentido se destacan los trabajos de Mekhalfa [2], Sholenikov [3], Nenov [4], Němček [5] y Goldfarb [6] en el dominio de las transmisiones por engranajes cilíndricos y de Kolotov [7], Nenov [8] y Timofieyev [9], en la síntesis de engranajes planetarios. Sin embargo, en estas aplicaciones, la obtención del contorno se realiza de manera gráfica. Es decir, el diseñador tiene que medir con las herramientas del software la coordenada que desea obtener. Asimismo, tampoco se tienen en cuenta las tolerancias de fabricación de las dimensiones de las ruedas en la construcción del contorno por lo que la zona de existencia de la transmisión no es del todo exacta.

El objetivo de esta investigación es perfeccionar el método de los Contornos de Bloqueo, desarrollando un modelo matemático, que permita la construcción no interactiva del contorno, considerando además la forma en que influyen las tolerancias del diámetro exterior, la distancia entre centros y la holgura lateral entre los perfiles de los dientes de las ruedas conjugadas, en cada una de las limitaciones geométricas. De esta manera, los valores de la zona de existencia de la transmisión resultan más exactos y pueden ser utilizados de manera analítica, sin la intervención del diseñador de engranajes.

\section{CONTENIDO}

\section{A. MATERIALES Y MÉTODOS}

1. Principales expresiones para la construcción automatizada y no interactiva del Contorno de Bloqueo

Para la construcción no interactiva del Contorno de Bloqueo se necesita transformar las expresiones de cada una de las limitaciones geométricas, de manera que sea posible su automatización. En esta investigación ello se realiza solucionando las ecuaciones $1,4,6$ y 7 en función de $x_{1}$, 
mientras que el valor de $\mathrm{x}_{2}$ se obtiene por la ecuación 8. Así, los valores mínimos $\left(\mathrm{x}_{1 \text { Mínimo }} \varepsilon_{\propto}\right)$ y máximos $\left(\mathrm{x}_{1 \text { Máximo }} \varepsilon_{\propto}\right)$ del coeficiente de corrección del piñón, que garantizan el factor de recubrimiento mínimo se obtienen por las siguientes ecuaciones:

$$
\begin{aligned}
& \mathrm{x}_{1 \text { Mínimo }} \varepsilon_{\propto}=-\frac{1}{2 \mathrm{a}_{2}}\left(\mathrm{a}_{3}+\sqrt{\mathrm{a}_{3}^{2}-4 \mathrm{a}_{2} \cdot \mathrm{a}_{4}}\right) \\
& \mathrm{x}_{1 \text { Máximo }} \varepsilon_{\propto}=\frac{1}{2 \mathrm{a}_{2}}\left(\sqrt{\mathrm{a}_{3}^{2}-4 \mathrm{a}_{2} \cdot \mathrm{a}_{4}}-\mathrm{a}_{3}\right)
\end{aligned}
$$

Donde:

$$
\begin{aligned}
& \mathrm{a}_{1}=\tan \left(\propto_{\mathrm{wt}}\right)\left(\mathrm{do}_{1}+\mathrm{do}_{2}\right)+\frac{2 \pi \cdot \varepsilon_{\alpha \text { Mínimo }} \cdot \cos \left(\alpha_{\mathrm{t}}\right)}{\cos (\beta)} \\
& \mathrm{a}_{2}=\left(\mathrm{cda}_{1}+\mathrm{cda}_{2}\right)^{2}-16 \mathrm{a}_{1}^{2} \\
& \mathrm{a}_{3}=8\left(\mathrm{cda}_{1}-\mathrm{cda}_{2}\right)^{3}-32 \mathrm{cda}_{1} \cdot \mathrm{cda}_{2}^{2}+32 \mathrm{cda}_{1}^{2} \cdot \mathrm{cda}_{2} \\
& +8\left(\mathrm{cda}_{1}+\mathrm{cda}_{2}\right)\left(\mathrm{do}_{2}^{2}-\mathrm{do}_{1}^{2}\right) \\
& -8 \mathrm{a}_{1}^{2}\left(\mathrm{cda}_{1}-\mathrm{cda}_{2}\right) \\
& \mathrm{a}_{4}=\mathrm{a}_{1}^{4}+\left(\left(\mathrm{cda}_{2}^{2}-\mathrm{cda}_{1}^{2}\right)+\left(\mathrm{do}_{1}^{2}-\mathrm{do}_{2}^{2}\right)\right)^{2}+2 \mathrm{a}_{1}^{2}\left(\mathrm{do}_{1}^{2}+\mathrm{do}_{2}^{2}\right) \\
& -2 \mathrm{a}_{1}^{2}\left(\mathrm{cda}_{2}^{2}+\mathrm{cda}_{1}^{2}\right) \\
& \operatorname{cda}_{1}=\frac{\cos \left(\alpha_{\mathrm{t}}\right)\left(\mathrm{z}_{1}+\mathrm{z}_{2}\right)}{\cos (\beta) \cos \left(\alpha_{\mathrm{wt}}\right)}-\frac{\mathrm{z}_{2}}{\cos (\beta)}-2 \mathrm{x}_{\mathrm{s}}+2 \mathrm{ha}^{*} \\
& \operatorname{cda}_{2}=\frac{\cos \left(\alpha_{t}\right)\left(z_{1}+z_{2}\right)}{\cos (\beta) \cos \left(\alpha_{w t}\right)}-\frac{z_{1}}{\cos (\beta)}+2 h^{*}
\end{aligned}
$$

El coeficiente de corrección mínimo, que garantiza la no interferencia entre los perfiles de las ruedas, se determina por la siguiente ecuación:

$\mathrm{x}_{1 \text { MínimoInterferencia }}=\frac{-\mathrm{b}_{2}+\sqrt{\mathrm{b}_{2}^{2}-4 \mathrm{~b}_{3}}}{2}$

Donde:

$\mathrm{b}_{1}=\mathrm{do}_{2} \cdot \tan \left(\propto_{\mathrm{wt}}\right)+\mathrm{do}_{1} \cdot \tan \left(\propto_{\mathrm{wt}}\right)-\mathrm{do}_{2} \cdot \tan \left(\propto_{\mathrm{t}}\right)+2 \frac{\left(\mathrm{h}-\mathrm{x}_{\mathrm{s}}\right)}{\operatorname{sen}\left(\alpha_{\mathrm{t}}\right)}$

$b_{2}=\tan ^{2}\left(a_{t}\right)\left(\frac{b_{1}}{\operatorname{sen}\left(\alpha_{t}\right)}-\operatorname{cda}_{1}\right)$

$\mathrm{b}_{3}=\frac{\left(\mathrm{b}_{1}^{2}-\mathrm{cda}_{1}^{2}+\mathrm{do}_{1}^{2}\right) \tan ^{2}\left(\alpha_{\mathrm{t}}\right)}{4}$

Y el coeficiente de corrección máximo:

$\mathrm{x}_{1 \text { MáximoInterferencia }}=\frac{-\mathrm{c}_{2}-\sqrt{\mathrm{c}_{2}^{2}-4 \mathrm{c}_{3}}}{2}$

Donde:

$c_{1}=\left(\mathrm{do}_{2}+\mathrm{do}_{1}\right) \tan \left(\propto_{\mathrm{wt}}\right)-\mathrm{do} \mathrm{o}_{1} \tan \left(\propto_{\mathrm{t}}\right)+2 \frac{\mathrm{h}}{\operatorname{sen}\left(\alpha_{\mathrm{t}}\right)}$

$c_{2}=\tan ^{2}\left(\alpha_{\mathrm{t}}\right)\left(\mathrm{cda}_{2}-\frac{\mathrm{Fo}}{\operatorname{sen}\left(\alpha_{\mathrm{t}}\right)}\right)$

$c_{3}=\tan ^{2}\left(\alpha_{\mathrm{t}}\right) \frac{\left(\mathrm{Fo}^{2}-\mathrm{cda}_{2}^{2}+\mathrm{do}_{2}^{2}\right)}{4}$

Los coeficientes de corrección mínimo y máximo, que garantizan el espesor mínimo del diente en el diámetro exterior de las ruedas, no se pueden obtener por expresiones analíticas. Aquí es necesario utilizar métodos numéricos. Para ello, la expresión 6 se expresa en función de $\mathrm{x}_{1}$ por medio de las ecuaciones 5, 8 y 15. De esta manera, se obtienen las expresiones 16 y 17 que representan la variación del espesor del diente en función del coeficiente de corrección del piñón. Se utiliza el método de Newton para calcular $\mathrm{x}_{1 \text { máximo }} \mathrm{S}_{\mathrm{a} 1} \mathrm{y}$ $\mathrm{x}_{1 \text { mínimo }} \mathrm{S}_{\mathrm{a} 2}$, mientras que para calcular $\mathrm{x}_{1 \text { mínimo }} \mathrm{S}_{\mathrm{a} 1}$ y $\mathrm{x}_{1 \text { máximo }} \mathrm{S}_{\mathrm{a} 2}$ se aplica el método de Hamming.

$$
\mathrm{da}_{1,2}=\mathrm{cda}_{1,2} \pm 2 \mathrm{x}_{1}
$$

$$
\begin{aligned}
\operatorname{Sa}_{1}\left(\mathrm{x}_{1}\right)=\left(\operatorname{cda}_{1}+\right. & \left.2 \mathrm{x}_{1}\right) \operatorname{Cos}(\beta)\left(\frac{\frac{\pi}{2}+2 \mathrm{x}_{1} \operatorname{Tan}\left(\alpha_{\mathrm{t}}\right)}{\mathrm{z}_{1}}\right. \\
& +\operatorname{Inv}\left(\propto_{\mathrm{t}}\right)-\frac{\sqrt{\left(\mathrm{cda}_{1}+2 \mathrm{x}_{1}\right)^{2}-\mathrm{do}_{1}^{2}}}{\mathrm{do}_{1}} \\
& \left.+\tan ^{-1}\left(\frac{\sqrt{(2.37)}}{\left.\mathrm{d} \operatorname{cda}_{1}+38\right)}\right)\right)
\end{aligned}
$$

$$
\begin{aligned}
& \operatorname{Sa}_{2}\left(x_{1}\right) \\
& =\left(\operatorname{cda}_{2}-2 x_{1}\right) \operatorname{Cos}(\beta)\left(\frac{\frac{\pi}{2}+\left(2 x_{s}-2 x_{1}\right) \operatorname{Tan}\left(\propto_{t}\right)}{z_{2}}\right. \\
& +\operatorname{Inv}\left(\propto_{t}\right)-\frac{\sqrt{\left(\operatorname{cda}_{2}-2 x_{1}\right)^{2}-d_{0}^{2}}}{d o_{2}} \\
& \left.+\tan ^{-1}\left(\frac{\sqrt{\left(\operatorname{cda}_{2}-2 x_{1}\right)^{2}-d_{0}^{2}}}{d o_{2}}\right)\right)
\end{aligned}
$$

Así, para un valor de corrección sumaria definido, se obtiene el

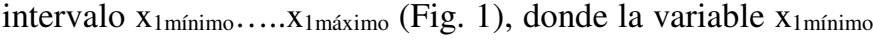
es el mayor de los valores de $\mathrm{x}_{1}$ calculados por las ecuaciones $1,9,13,16$ y 17 . Asimismo, el valor de $\mathrm{x}_{1 \text { máximo }}$ es el menor de los valores máximos de $\mathrm{x}_{1}$ calculados por las ecuaciones 1,10 , 14,16 y 17.

La obtención del contorno se reduce entonces a calcular el valor de corrección sumaria por la ecuación 18, luego de asignar un valor al ángulo de presión en el plano frontal. El cálculo comienza en $\propto_{\mathrm{wt}}=1^{\mathrm{o}}$ y termina en $\propto_{\mathrm{wt}}=45^{\circ}[1]$. El valor de $\propto_{\mathrm{wt}}$ se aumenta cada vez en un grado. La precisión de los valores del ángulo de presión $\left(\propto_{\text {wtMínimo } \mathrm{y}} \propto_{\text {wtMáximo }}\right)$ correspondientes a los valores de corrección sumaria mínimo y máximo se mejora utilizando el método numérico de la bisección.

$\mathrm{x}_{\mathrm{S}}=\frac{\left(\operatorname{inv}\left(\alpha_{\mathrm{wt}}\right)-\operatorname{inv}\left(\alpha_{\mathrm{t}}\right)\right)}{2 \tan \left(\alpha_{\mathrm{t}}\right)}\left(\mathrm{z}_{1}+\mathrm{z}_{2}\right)$

La metodología expuesta permite establecer la relación geométrica entre las variables que definen una transmisión por engranajes cilíndricos con contacto exterior, mediante la utilización analítica del método de los Contornos de Bloqueo. Así, por ejemplo, por la ecuación 19 se obtienen los valores mínimo y máximo de la distancia entre centros para módulo unitario $\left(\mathrm{a}_{\text {wmínimo_m }=1} \mathrm{y} \mathrm{a}_{\text {wmáximo_m }}\right.$ ) , utilizando los valores mínimo y máximo del ángulo de presión. De esta manera, la expresión 20 establece la manera exacta en que se relaciona el módulo y la distancia entre centros para una combinación definida de los parámetros de la herramienta, números de dientes de las ruedas y el ángulo de inclinación del diente.

$\mathrm{a}_{\mathrm{w}}=\frac{\mathrm{m}\left(\mathrm{z}_{1}+\mathrm{z}_{2}\right)}{2 \operatorname{Cos}(\beta)}\left(\frac{\cos \left(\alpha_{\mathrm{t}}\right)}{\cos \left(\alpha_{\mathrm{wt}}\right)}\right)$

$\mathrm{a}_{\text {wmínimo_m }=1} \leq \frac{\mathrm{a}_{\mathrm{w}}}{\mathrm{m}} \leq \mathrm{a}_{\text {wmáximo_m }=1}$

Sin embargo, la correlación puramente geométrica entre las variables que definen una transmisión por engranajes puede producir resultados no totalmente correctos. Por ejemplo, para los siguientes parámetros geométricos: $\mathrm{z}_{1}=10, \mathrm{z}_{2}=60, \beta=$ $0^{\circ}, m=1, \mathrm{a}_{\mathrm{w}}=35 \mathrm{~mm}$, el modelo matemático descrito indica 
los siguientes valores limites de los coeficientes de corrección: desde $\mathrm{x}_{1 \text { Mínimo }}=0.402 \mathrm{y} \mathrm{x}_{2 \text { Máximo }}=-0.402$ hasta $\mathrm{x}_{1 \text { Máximo }}=$ 0.444 y x 2 Mínimo $=-0.444$ (Fig. 2).

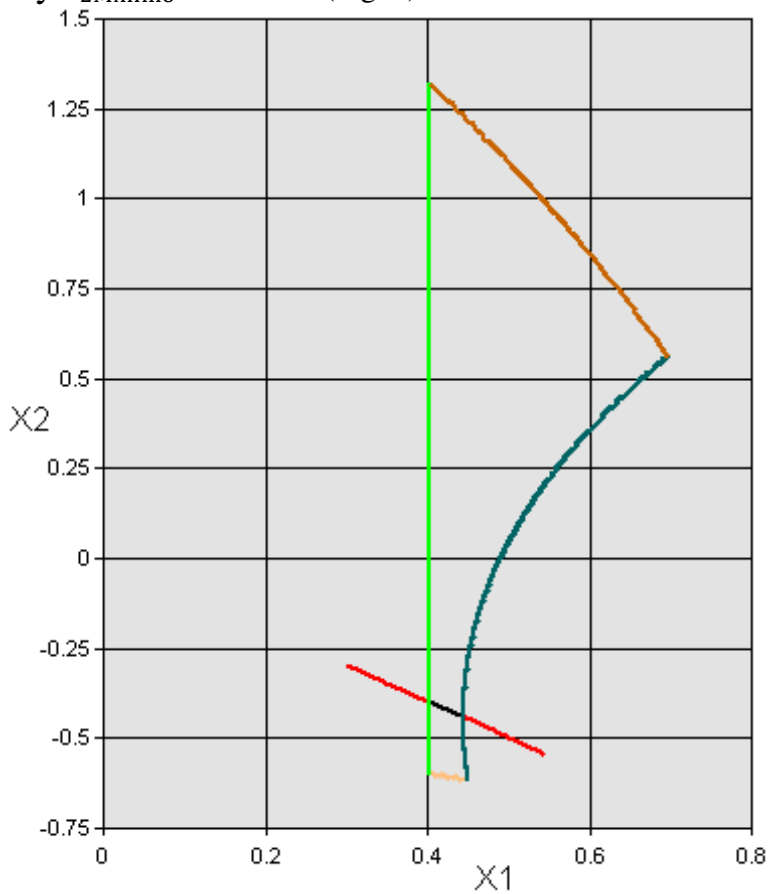

Figura 2. Contorno de Bloqueo para $\mathrm{z}_{1}=10, \mathrm{z}_{2}=60, \beta=0^{\circ}$, $\mathrm{Sa}_{1,2 \text { Mínimo }}=0,25, \varepsilon_{\propto \text { Mínimo }}=1,2$

Factor de recubrimiento

Socavado

Espesor del diente en el diámetro exterior del piñón Interferencia

Segmento válido para el valor dado de $\mathrm{x}_{\mathrm{S}}$

Ello implica, que el intervalo donde se cumplen las limitaciones geométricas es el siguiente:

En el piñón:

$$
\mathrm{m}\left(\mathrm{x}_{1 \text { Máximo }}-\mathrm{x}_{1 \text { Mínimo }}\right)=1(0,444-0,402)=0,042 \mathrm{~mm}
$$

En la rueda:

$$
\mathrm{m}\left(\mathrm{x}_{2 \text { Máximo }}-\mathrm{x}_{2 \text { Mínimo }}\right)=1(-0,402+0,444)=0,042 \mathrm{~mm}
$$

Es decir, se observa que el intervalo valido es muy pequeño. Por otro lado, se conoce que las dimensiones de las ruedas se fabrican con determinadas tolerancias. Surge entonces la duda de si se mantiene o no el intervalo anterior con la variación de las dimensiones de las ruedas dentro del campo de tolerancia.

3. Influencia de la holgura lateral en los límites del contorno

La mayor afectación de los límites del contorno tiene lugar cuando la holgura lateral entre los dientes en contacto se obtiene con el máximo desplazamiento de la herramienta, en el

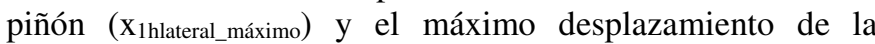
herramienta en la rueda ( $\mathrm{x}_{2}$ hlateral_máximo). Para garantizar que los valores de $\mathrm{x}_{1} \mathrm{y}_{\mathrm{x}_{2}}$ encerrados por los límites del contorno garanticen la ausencia de socavado en ambas ruedas se deben desplazar hacia el interior del mismo las rectas 5 y 6 en las magnitudes $\mathrm{x}_{\text {1hlateral_máximo } \mathrm{y}} \mathrm{x}_{2 \text { hlateral_máximo }}$ respectivamente, como se muestra en la Fig. 3.

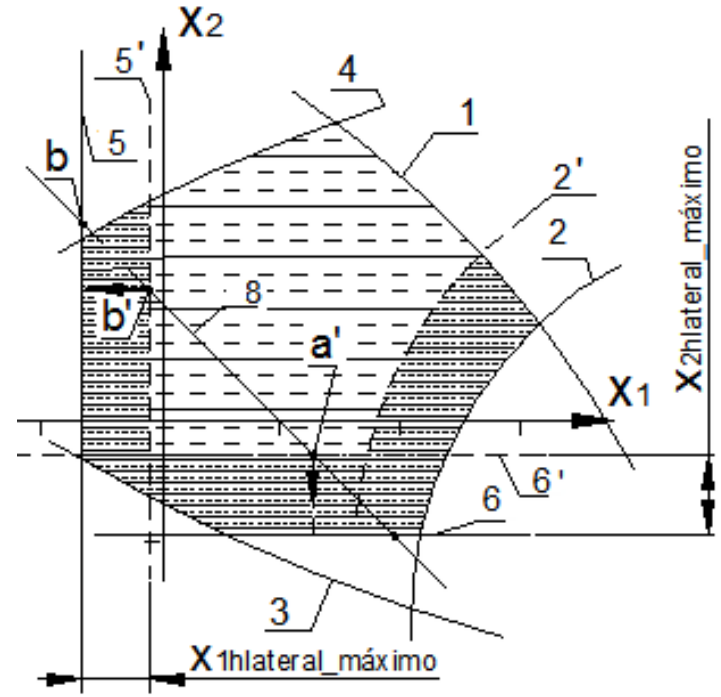

Figura 3. Influencia de la holgura lateral en el contorno

Lo anterior se expresa matemáticamente modificando la ecuación 1 de la siguiente manera:

$$
\mathrm{x}_{1,2} \geq \mathrm{h}-\frac{\mathrm{z}_{1,2} \operatorname{sen}^{2}\left(\alpha_{\mathrm{t}}\right)}{2 \cos (\beta)}+\mathrm{x}_{1,2 \text { hlateral_máximo }}
$$

También es necesario trasladar hacia el interior del contorno las curvas que limitan el espesor del diente (curva 2' en la Fig. 3). En este caso, la nueva posición se determina modificando las ecuaciones 16 y 17 como se muestra a continuación:

$$
\begin{aligned}
& \mathrm{Sa}_{1}\left(\mathrm{x}_{1}\right)=\left(\mathrm{cda}_{1}+2 \mathrm{x}_{1}\right) \operatorname{Cos}(\beta) \\
& \left(\frac{\frac{\pi}{2}+2\left(\mathrm{x}_{1}-\mathrm{x}_{1 \text { hlateral }_{\text {máximo }}}\right) \operatorname{Tan}\left(\propto_{\mathrm{t}}\right)}{\mathrm{z}_{1}}+\operatorname{Inv}\left(\propto_{\mathrm{t}}\right)\right. \\
& -\frac{\sqrt{\left(\mathrm{cda}_{1}+2 \mathrm{x}_{1}\right)^{2}-\mathrm{do}_{1}^{2}}}{\mathrm{do}_{1}} \\
& \left.+\tan ^{-1}\left(\frac{\sqrt{\left(\mathrm{cda}_{1}+2 \mathrm{x}_{1}\right)^{2}-\mathrm{do}_{1}^{2}}}{\mathrm{do}_{1}}\right)\right) \\
& \mathrm{Sa}_{2}\left(\mathrm{x}_{1}\right)=\left(\mathrm{cda}_{2}-2 \mathrm{x}_{1}\right) \operatorname{Cos}(\beta) \\
& \left(\frac{\frac{\pi}{2}+2\left(\mathrm{x}_{\mathrm{s}}-\mathrm{x}_{\text {2hlateral }_{\text {máximo }}}-\mathrm{x}_{1}\right) \operatorname{Tan}\left(\propto_{\mathrm{t}}\right)}{\mathrm{z}_{2}}+\operatorname{Inv}\left(\propto_{\mathrm{t}}\right)\right. \\
& -\frac{\sqrt{\left(\mathrm{cda}_{2}-2 \mathrm{x}_{1}\right)^{2}-\mathrm{do}_{2}^{2}}}{\mathrm{do}_{2}} \\
& \left.+\tan ^{-1}\left(\frac{\sqrt{\left(\mathrm{cda}_{2}-2 \mathrm{x}_{1}\right)^{2}-\mathrm{do}_{2}^{2}}}{\mathrm{do}_{2}}\right)\right)
\end{aligned}
$$

La holgura lateral favorece la no interferencia entre los perfiles ya que aumenta la altura del diente con perfil evolvente, mientras que sobre el factor de recubrimiento no ejerce ninguna influencia.

4. Influencia de la tolerancia de fabricación del diámetro exterior en los límites del contorno 
Al analizar la Fig. 4 y las ecuaciones 4 y 5 , se puede concluir que el menor valor del factor de recubrimiento, se obtiene cuando los diámetros exteriores de ambas ruedas tienen el valor mínimo.

En este caso, la curva que representa esta limitación geométrica también se traslada hacia el interior del contorno.

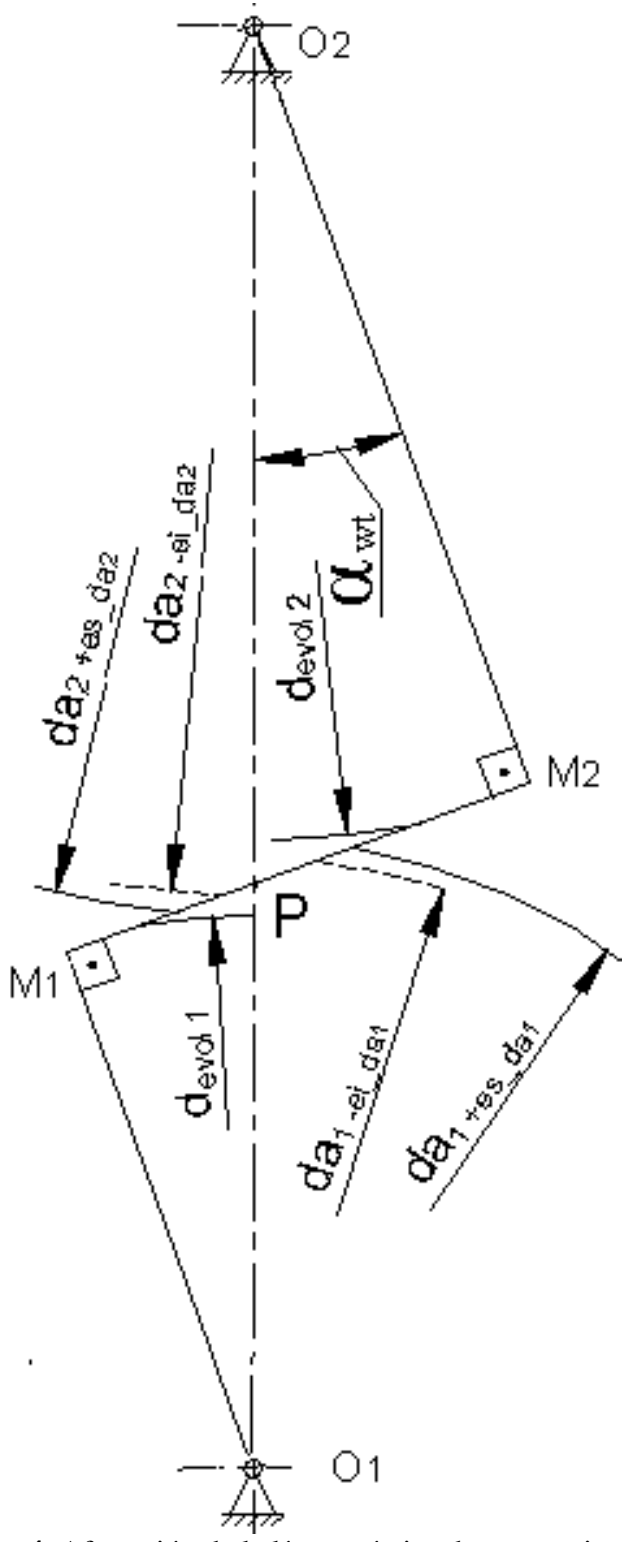

Figura 4. Afectación de la línea práctica de engranaje por la tolerancia del diámetro exterior

es_da $\mathrm{da}_{1,2} \rightarrow$ Desviación superior del diámetro exterior del piñón y la rueda respectivamente.

ei_da $\mathrm{d}_{1,2} \rightarrow$ Desviación inferior del diámetro exterior del piñón y la rueda respectivamente.

$\mathrm{d}_{\text {evol1,2 }} \rightarrow$ Diámetro de inicio del perfil evolvente en el piñón y la rueda respectivamente.

La nueva posición se obtiene modificando las ecuaciones 11 y 12 de la manera siguiente:

$$
\begin{aligned}
& \operatorname{cda}_{1}=\frac{\cos \left(\alpha_{t}\right)\left(z_{1}+z_{2}\right)}{\cos (\beta) \cos \left(\alpha_{w t}\right)}-\frac{z_{2}}{\cos (\beta)}-2 x_{s}+2 h^{*}-\text { ei_da } \\
& \operatorname{cda}_{2}=\frac{\cos \left(\alpha_{t}\right)\left(z_{1}+z_{2}\right)}{\cos (\beta) \cos \left(\alpha_{w t}\right)}-\frac{z_{1}}{\cos (\beta)}+2 h^{*}-\text { ei_da } 2
\end{aligned}
$$

De manera inversa sucede, en el caso del espesor del diente y la interferencia, donde la mayor afectación en los límites del contorno se obtiene con los diámetros exteriores máximos. En este caso, se necesita modificar las ecuaciones 11 y 12 como se indica a continuación:

$\operatorname{cda}_{1}=\frac{\cos \left(\alpha_{t}\right)\left(z_{1}+z_{2}\right)}{\cos (\beta) \cos \left(\alpha_{w t}\right)}-\frac{z_{2}}{\cos (\beta)}-2 x_{s}+2 h a^{*}+$ es_da
$\operatorname{cda}_{2}=\frac{\cos \left(\alpha_{t}\right)\left(z_{1}+z_{2}\right)}{\cos (\beta) \cos \left(\alpha_{w t}\right)}-\frac{z_{1}}{\cos (\beta)}+2 h^{*}+$ es_da

Se debe destacar que el socavado no tiene ninguna dependencia de las dimensiones exteriores de las ruedas.

5. Influencia de la tolerancia de la distancia entre centros en los límites del contorno

La precisión de fabricación de la distancia entre centros modifica el ángulo de presión de la transmisión. Cuando la distancia entre centros tiene el valor mínimo, también es mínimo el ángulo de presión (Fig. 5) y viceversa.

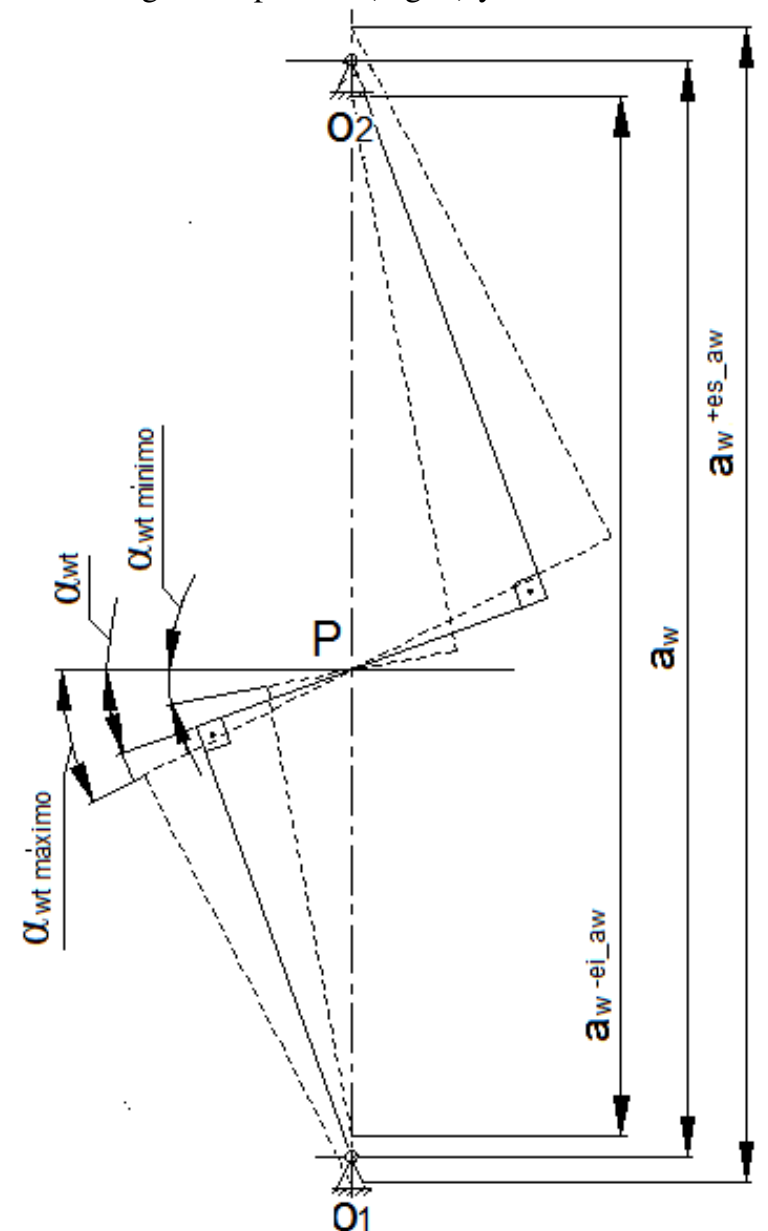

Figura 5. Variación del ángulo de presión por la tolerancia de la distancia entre centros

es_a $a_{w} \rightarrow$ Desviación superior de la distancia entre centros. ei_a $\mathrm{a}_{\mathrm{w}} \rightarrow$ Desviación inferior de la distancia entre centros. Los ángulos mínimo y máximo se calculan por la siguiente expresión: 
28

$$
\propto_{\mathrm{wT}_{\text {Minimo }}^{\text {Maximo }}}=\cos ^{-1}\left(\frac{\mathrm{a}_{\mathrm{w}} \cos \left(\alpha_{\mathrm{wt}}\right)}{\mathrm{a}_{\mathrm{w}}{ }_{-\mathrm{es} \mathrm{s}_{\mathrm{w}} \mathrm{a}_{\mathrm{w}}}}\right)
$$

Scientia et Technica Año XXI, Vol. 21, No. 1, marzo de 2016. Universidad Tecnológica de Pereira.

Del análisis de la ecuación 4 y la Fig. 5 se puede concluir que la mayor afectación a la curva del factor de recubrimiento se tiene cuando es máximo el ángulo de presión $\left(\alpha_{\text {wrMáximo }}\right)$. En el caso de la interferencia sucede exactamente lo contrario. Aquí, al disminuir el ángulo de presión, aumenta la probabilidad de que la circunferencia del diámetro exterior del diente corte la línea de engranaje en un punto situado por debajo del diámetro de inicio del perfil evolvente de la rueda conjugada. Las limitaciones geométricas de socavado y espesor del diente no son afectadas por la tolerancia de fabricación de la distancia entre centros.

\section{B. RESULTADOS Y DISCUSIÓN}

En la Fig. 6 se muestran los Contornos de Bloqueo obtenidos considerando el grado de precisión (interior) y de la forma tradicional (exterior) para las siguientes condiciones:

- $\mathrm{z}_{1}=35$ y z $\mathrm{z}_{2}=64$

- Parámetros de la herramienta: $\mathrm{ha}^{*}=1 ; \mathrm{c}^{*}=0,25$; $\rho^{*}=0,4 ; \propto=20^{\circ}$

- Grado de precisión cinematica y de suavidad de la marcha igual a 8 [10].

- Precisión del diámetro exterior h7.

- Tipo de holgura lateral B [10].

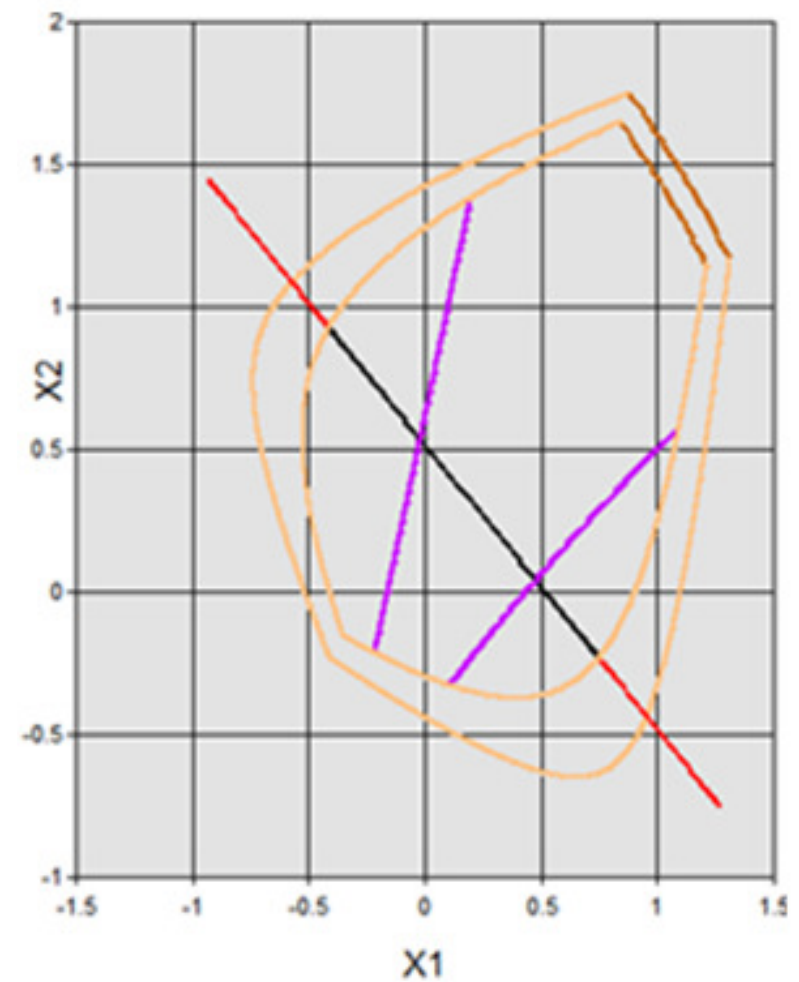

Figura 6. Contorno de Bloqueo para $z_{1}=20, z_{2}=30, \beta=$ $10,844^{\circ}, \mathrm{m}=5,5 \mathrm{~mm}$,

$\mathrm{Sa}_{1,2 \text { Mínimo }}=0,25 \mathrm{~m}=0,25 \cdot 5,5=1,38 \mathrm{~mm}, \varepsilon_{\propto \text { Mínimo }}=1,2$

Curvas que limitan las zonas de contacto de dos pares de dientes en el polo de engranaje

Se ha introducido además en el área gráfica la recta que corresponde a una transmisión con $\mathrm{m}=3 \mathrm{~mm} \mathrm{y} \mathrm{a}_{\mathrm{w}}=150 \mathrm{~mm}$. El color negro representa la zona de valores validos de $\mathrm{x}_{1} \mathrm{y} \mathrm{x}_{2}$. Asimismo el color rojo indica las combinaciones de $\mathrm{x}_{1} \mathrm{y}_{2}$ que se encuentra fuera del contorno.

Aquí se destaca que la figura tiene solamente una función ilustrativa ya que el diseñador no interactúa con la imagen para obtener el resultado de la construcción del contorno. Este se utiliza directamente para realizar los cálculos necesarios o se muestra en un formulario para este fin.

Se debe destacar además, que los valores de las tolerancias para todo el contorno, se toman a partir de las dimensiones de la transmisión de referencia, es decir con $\mathrm{x}_{1}=\mathrm{x}_{2}=0$. Por esta razón, a diferencia de la forma tradicional, el Contorno de Bloqueo construido teniendo en cuenta las tolerancias es dependiente del módulo de la transmisión.

En un análisis de las curvas que limitan el contorno (Fig. 6), se observa que el mismo está restringido por la interferencia y el factor de recubrimiento solamente. Los coeficientes de corrección ubicados en los bordes representan los valores límites que se pueden utilizar en correspondencia con las tolerancias seleccionadas para la holgura lateral, el diámetro exterior y la distancia entre centros. De esta manera se garantiza, que aun con las combinaciones más desfavorables de las tolerancias, se cumple con las restricciones geométricas de socavado, interferencia, aguzamiento del diente y factor de recubrimiento, lo que indudablemente mejora la calidad del diseño obtenido.

La corrección sumaria, necesaria para insertar la transmisión en la distancia entre centros de $150 \mathrm{~mm}$ es de 0,518 (Fig. 6). Los coeficientes de corrección límites son:

$$
\mathrm{x}_{1 \text { Mínimo }}=-0,41 \quad \mathrm{y} \quad \mathrm{x}_{2 \text { Máximo }}=0,928
$$

La selección definitiva de los coeficientes de corrección se realiza teniendo en cuenta las condiciones reales de trabajo de la transmisión. En ese sentido, algunos autores [1] recomiendan utilizar en transmisiones cerradas, cuando la distancia entre centros está restringida a valores normalizados, los coeficientes de corrección que garantizan dos pares de dientes en contacto en el polo de engranaje.

En la Fig. 6 se han introducido en el área gráfica estas curvas. Se observa, que para la transmisión con $\mathrm{a}_{\mathrm{w}}=150 \mathrm{~mm}$, existen dos intervalos donde se puede garantizar el contacto entre dos pares de dientes en el polo de engranaje. De $\mathrm{x}_{1}=-0,41$, $\mathrm{x}_{2}=0,928$ limitado por la interferencia a $\mathrm{x}_{1}=-0,019, \mathrm{x}_{2}=0,537$ $\mathrm{y}$ de $\mathrm{x}_{1}=0,478, \mathrm{x}_{2}=-0,04$ a $\mathrm{x}_{1}=0,749, \mathrm{x}_{2}=-0,231$ limitado también por la interferencia.

Aunque ambos intervalos garantizan dos pares de dientes en el polo de engranajes, es evidente que no se debe utilizar el primero de ellos, ya que aquí el diente del piñón resulta debilitado por la corrección negativa. La solución buscada se toma entonces en el segundo intervalo. Aquí se destaca la importancia de la construcción del contorno teniendo en cuenta las tolerancias. Ello permite utilizar como solución definitiva los coeficientes de corrección que están en el borde mismo del contorno $\mathrm{x}_{1}=0,749, \mathrm{x}_{2}=-0,231$. Este punto corresponde a cuando el contacto entre un solo par de dientes comienza lo más alejado posible del polo de engranaje, garantizando además la no interferencia entre los perfiles, aun con la combinación más desfavorable de las tolerancias.

Otro resultado importante de la construcción del Contorno de Bloqueo teniendo en cuenta las tolerancias es en relación con 
los números de dientes. En la tabla 1 se muestran los números de dientes mínimos y máximos para diferentes normas de la cremallera de referencia y las siguientes condiciones:

- Grados de precisión cinematica y de suavidad de la marcha igual a 7 .

- Tolerancia del diámetro exterior h7.

- Tipo de holgura lateral C.

- $\quad \varepsilon_{\propto \text { Mínimo }}=1,2$

- $\mathrm{m}=5 \mathrm{~mm}$

- $\beta=0^{\circ}$

- $\mathrm{Sa}_{1,2 \text { Mínimo }}=0,25 \cdot \mathrm{m}=0,25 \cdot 5=1,25 \mathrm{~mm}$

Tabla 1. Combinaciones mínima y máxima de z1 y z2 obtenidas sin tener en cuenta las tolerancias de fabricación.

\begin{tabular}{|c|c|c|c|c|c|c|c|}
\hline \multicolumn{4}{|c|}{ Combinación } & \multicolumn{4}{|c|}{ Herramienta } \\
\hline \multicolumn{2}{|c|}{ Mínima } & \multicolumn{2}{|c|}{ Máxima } & \multirow{2}{*}{ ha $^{*}$} & \multirow{2}{*}{$c^{*}$} & \multirow{2}{*}{$\rho^{*}$} & \multirow{2}{*}{$\begin{array}{l}\propto \\
\left({ }^{0}\right)\end{array}$} \\
\hline $\mathrm{Z}_{1}$ & $\mathrm{z}_{2}$ & $\mathrm{Z}_{1}$ & $\mathbf{Z}_{2}$ & & & & \\
\hline 12 & 12 & 1073 & 1073 & 1 & 0,25 & 0,4 & 20 \\
\hline 12 & 13 & 71 & 71 & 1 & 0,157 & 0,47 & 14,5 \\
\hline
\end{tabular}

Tabla 2. Combinaciones mínima y máxima de z1 y z2 obtenidas teniendo en cuenta las tolerancias de fabricación.

\begin{tabular}{|c|c|c|c|c|c|c|c|}
\hline \multicolumn{4}{|c|}{ Combinación } & \multicolumn{4}{|c|}{ Herramienta } \\
\hline \multicolumn{2}{|c|}{ Mínima } & \multicolumn{2}{|c|}{ Máxima } & \multirow{2}{*}{ ha $^{*}$} & \multirow{2}{*}{$c^{*}$} & \multirow{2}{*}{$\rho^{*}$} & \multirow{2}{*}{$\begin{array}{l}\propto \\
\left({ }^{0}\right)\end{array}$} \\
\hline $\mathrm{Z}_{1}$ & $\mathrm{z}_{2}$ & $\mathrm{Z}_{1}$ & $\mathrm{z}_{2}$ & & & & \\
\hline 12 & 13 & 284 & 284 & 1 & 0,25 & 0,4 & 20 \\
\hline 13 & 14 & 63 & 64 & 1 & 0,157 & 0,47 & 14,5 \\
\hline
\end{tabular}

En las figuras 7 y 8 se muestran los Contornos de Bloqueo para las combinaciones minima y maxima de los números de dientes y la herramienta: $\mathrm{ha}^{*}=1, \mathrm{c}^{*}=0,25, \rho^{*}=0,4, \propto=20^{\circ}$. Se observa que los números de dientes máximos estan limitados por la condición de interferencia solamente, mientras que los números de dientes mínimos estan limitados por el socavado y el factor de recubrimiento.

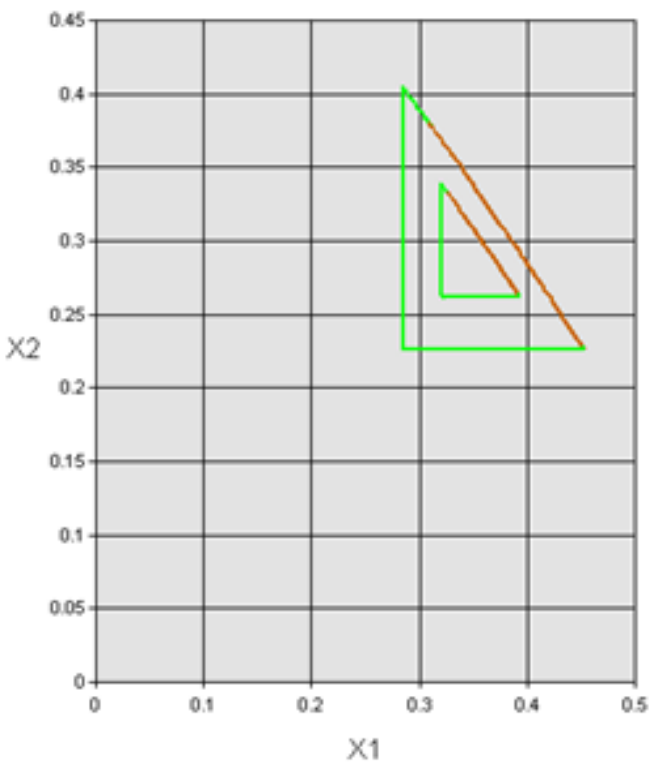

Figura 7. Contornos de Bloqueo para las combinaciones mínima y máxima de los números de dientes.
En este caso, los números de dientes mínimos y máximo son los valores límites, que aun con las combinaciones más desfavorables de las tolerancias, se pueden utilizar para la síntesis de transmisiones por engranajes cilíndricos con contacto exterior, garantizando el cumplimiento de las restricciones geométricas de socavado, interferencia, factor de recubrimiento y aguzamiento del diente.

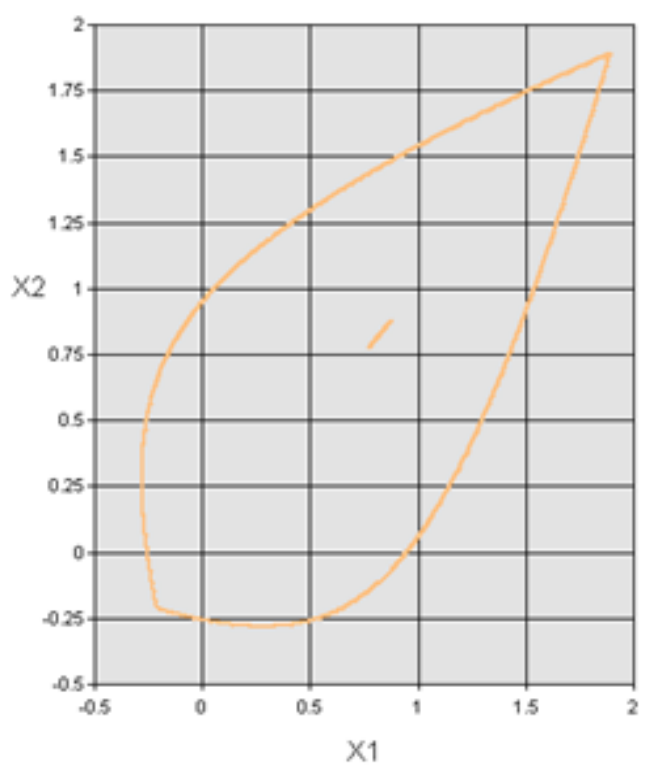

Figura 8. Contornos de Bloqueo para las combinaciones mínima y máxima de los números de dientes.

\section{CONCLUSIONES Y RECOMENDACIONES}

El método de los Contornos de Bloqueo se utiliza en la actualidad de manera automatizada. Se reportan aplicaciones para la síntesis de transmisiones planetarias y transmisiones por engranajes cilíndricos con contacto exterior e interior, sin embargo no se constató en la literatura especializada, la reducción de los límites del contorno considerando las tolerancias de fabricación de las ruedas.

La construcción del Contorno de Bloqueo, teniendo en cuenta las tolerancias del diámetro exterior, de la distancia entre centros y de la holgura lateral, permite obtener la zona valida de los coeficientes de corrección de una manera más perfeccionada. De esta manera, se garantiza que todas las combinaciones de $\mathrm{x}_{1} \mathrm{y} \mathrm{x}_{2}$ limitados por los bordes del contorno, cumplen con las limitaciones geométricas de socavado, factor de recubrimiento, espesor del diente en el diámetro exterior e interferencia, aun con las combinaciones más desfavorables de las tolerancias.

\section{REFERENCIAS}

[1]. Bolotovski, I. A. Bezrukov, O. F. Vacilieva, B. I. Cálculo Geométrico de Transmisiones de Perfil Evolvente por Engranajes y por Tornillo Sin Fin. Original en Ruso, 2da Edición, Moscú, Construcción de Maquinarias 1986, 444 p.p. 28-138. ISBN 5-0689717 -A 
[2]. Mekhalfa, A. Hadjadj, E. Kallouche, A. Cylindrical Teeth's Gear in Devellopante of Circle. Journal of Engineering and Applied Sciences [en línea] vol. 2, $\mathrm{n}^{\circ} 1 \mathrm{p}$. 121-124, 2007 [Consultado el 15 de Diciembre 2013]. Disponible http://medwelljournals.com/abstract/?doi=jeasci.2007.121 .124 ISSN : 1818-7803

[3]. Sholeninov, V.E. Hikishin, S. M. Síntesis de Engranajes Cilíndricos con Ayuda de la Computadora (En Ruso) Vestnik D.G.M.A. [En línea] vol. 14, no 3E, p. 187-191, 2008 [Consultado el 15 de Diciembre 2013]. Disponible en: http://opm.donntu.edu.ua/files/sbornik/dm2008.pdf ISSN 1995-4794

[4]. Nenov, P. Ronkova, V. Angelova, E., et al. Almanac of Information Materials, 3d Images, Animations and other Visualizations as an Aid in Studying Machine Elements, en The 7th International Conference Research and Development of Mechanical Elements and Systems, Zlatibor, Serbia 2011, p.119-126 [Consultado el 15 de Diciembre 2013]. Disponible en: http://phdproject.uni-ruse.bg/files/article/paperIRMES2011-BGRUSE.pdf ISBN 978-86-6055-012-7

[5]. Němček, M. Design of a non Standard Internal Gear Pair. Transactions of the VSB-Technical University of Ostrava [En linea] vol LII, no 1 p. 141-145, 2007 [Consultado el 15 de Diciembre 2013]. Disponible en: http://transactions.fs.vsb.cz/2007-1/1541.pdf , ISSN 12100471

[6]. Goldfarb, V. I. Tkachev, A. A. Optimization Approach to Computer-Aided Design of Spur and Helical Gears, en: 13th World Congress in Mechanism and Machine Science, Guanajuato, México, 2011, p.1-6 [Consultado el 15 de Diciembre

Disponible

2013].

http://somim.org.mx/conference_proceedings/area.html

ISBN 978-607-441-131-7

[7]. Kolotov, A. V. Merko, V. A. Mesnyankin, M. V., et al. Resultados de la Solución del Problema de la Determinación de la Zona Común de Selección de los Coeficientes de Desplazamiento de la Transmisión por Engranajes del Tipo 2K-h (En Ruso). Viestnik de la Universidad Técnica de Tadzhikistan [En línea] $\mathrm{N}^{\mathrm{o}} 2$, vol. 22, p. 26-29, 2013 [Consultado el 15 de Diciembre 2013]. Disponible http://www.ttu.tj/userfiles/vestnik/vn22.pdf ISSN 2075$177 \mathrm{X}$

[8]. Nenov, P. Kaloyanov, B. Angelova, E. Geometrical Blocking Contours as an Instrument for High-Technology Design of Geometry of Involute External and Internal Gear Drives. Mechanisms and Machine Science [En linea] Vol. 13, p. 379-388, 2013 [Consultado el 15 de Diciembre 2013]. Disponible en: http://link.springer.com/chapter/10.1007/978-94-0076558-0_29\#page-1 ISSN 0094-114X

[9]. Timofieyev, G. A. Samailova, M. V. Zona de existencia de un Mecanismo Planetario de Onda" (En Ruso). Viestnic MGTU Bauman H E Serie: Construcción de Maquinaria [En línea] $\mathrm{N}^{\mathrm{o}}$ 2, p. 117-122, 2012 [Consultado el 15 de Diciembre 2013]. Disponible en: http://vestnikmach.bmstu.ru/articles/98/98.pdf $\quad$ ISSN 0236-3941 